\title{
Charge Regulation as a Stabilization Mechanism for Shell-Like Assemblies of Polyoxometalates
}

\author{
Aletta A. Verhoeff, ${ }^{1}$ Melissa L. Kistler, ${ }^{2}$ Anish Bhatt, ${ }^{2}$ Joe Pigga, ${ }^{2}$ Jan Groenewold, ${ }^{1}$ Mark Klokkenburg, ${ }^{1}$ Sandra Veen, ${ }^{1}$ \\ Soumyajit Roy, ${ }^{1}$ Tianbo Liu, ${ }^{2, *}$ and Willem K. Kegel ${ }^{1, *}$ \\ ${ }^{1}$ Van 't Hoff Laboratory for Physical and Colloid Chemistry, Debye Institute, Utrecht University, Padualaan 8, 3584 CH Utrecht, \\ The Netherlands \\ ${ }^{2}$ Dept. of Chemistry, Lehigh University, 6 E Packer Ave, Bethlehem, Pennsylvania 18015, USA
}

(Received 21 August 2006; published 8 August 2007)

\begin{abstract}
We show that the equilibrium size of single-layer shells composed of polyoxometalate macroions is inversely proportional to the dielectric constant of the medium in which they are dispersed. This behavior is consistent with a stabilization mechanism based on Coulomb repulsion combined with charge regulation. We estimate the cohesive energy per bond between macroions on the shells to be approximately $-6 k T$. This number is extracted from analysis based on a charge regulation model in combination with a model for defects on a sphere. The value of the cohesive bond energy is in agreement with the model-independent critical aggregate concentration. This observation points to a new class of thermodynamically stable shell-like objects. We point out the possible relevance our findings have for certain surfactant systems.
\end{abstract}

DOI: 10.1103/PhysRevLett.99.066104

PACS numbers: 82.45.Mp, 73.30.+y, 89.75.Fb

Recently, it has been demonstrated that building blocks made of (mainly) metal oxide polyhedrals (molybdenum, tungsten, vanadium, iron, etc.) may lead to intricate, highly symmetrical molecular structures composed of hundreds of metal oxide monomers by sharing oxygen atoms, see, e.g., Ref. [1] for a review, and Fig. 1 (left) for an illustration. These clusters, representing some of the largest inorganic molecules, are referred to as polyoxometalates or POMs. Also quite recently, it was shown that POMs, in turn, may spontaneously and reversibly form superstructures: hollow, spherical objects with an average, almost monodisperse radius in water of several tens of nanometers, and composed of more than 1000 of individual POM macroions [2], as illustrated in Fig. 1. Not only molybdenum-containing POMs form these intriguing shell-like superstructures, but also those containing tungsten and copper [3]. We will further refer to the shell-like superstructures as "shells" or "POM-shells." Strong evidence for the hollow, single-layer structure of the shells comes from light scattering studies and is backed up with electron microscopy [2].

At this point, it is an open question why these shells are thermodynamically stable. The question of stability is twofold. First of all, why do the POMs form single-layer structures? The formation of a single-layer structure seems to be incompatible with the spherically symmetric shape of (some of) the POMs. To date, this paradox has not been resolved and remains so in this Letter. The question we address here is: Given the existence of the single-layer structure in the form of a shell, what determines its size? In other words, why do not shells grow into an infinite structure? This way the single-layer structure requires a minimum bending energy. As POM-shells are commonly studied under low ionic strength, Coulombic charges on the POMs may prevent growth into an infinite structure. This has motivated us to study equilibrium shell size as a function of dielectric constant.

We studied two C60-like, hollow, spherically shaped, but chemically different polyoxometalates, shown schematically in Fig. 1. These have the following composition in their crystalline state: $\quad\left(\mathrm{NH}_{4}\right)_{42}\left[\left(\mathrm{Mo}_{72}^{\mathrm{VI}} \mathrm{Mo}_{60}^{\mathrm{V}} \mathrm{O}_{372}\left(\mathrm{CH}_{3} \mathrm{COO}\right)_{30}\left(\mathrm{H}_{2} \mathrm{O}\right)_{72}\right]\right.$.ca. $300 \mathrm{H}_{2}$ O.ca. $10 \mathrm{CH}_{3} \mathrm{COONH}_{4}$, abbreviated as $\mathrm{Mo}_{132}$, and $\mathrm{Mo}_{72}^{\mathrm{VI}} \mathrm{Fe}_{30}^{\mathrm{III}} \mathrm{O}_{252} L_{102}$.ca. $180 \mathrm{H}_{2} \mathrm{O}$, with $L=\mathrm{H}_{2} \mathrm{O} /$ $\mathrm{CH}_{3} \mathrm{COO}^{-} / \mathrm{Mo}_{2} \mathrm{O}_{8 / 9}^{n-}$ and further referred to as

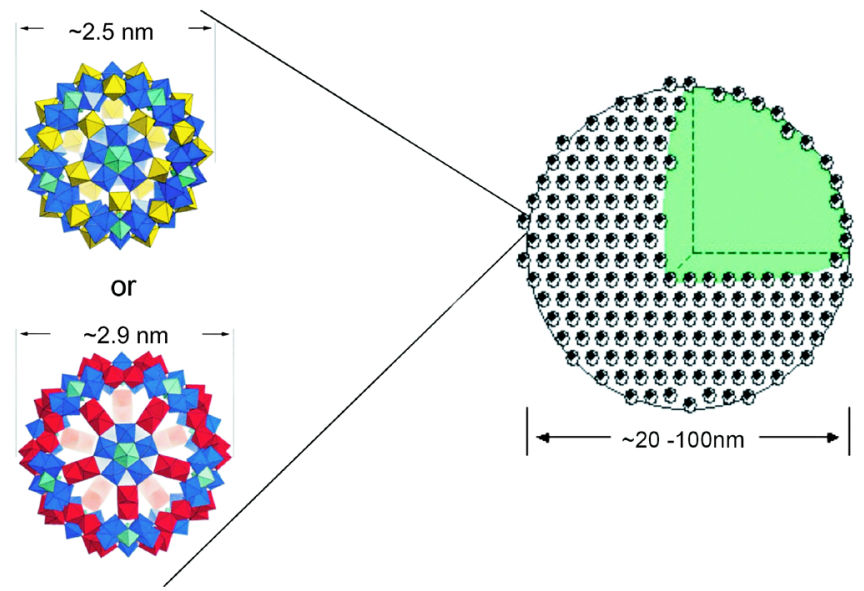

FIG. 1 (color online). Polyoxometalates (POMs) $\mathrm{Mo}_{72} \mathrm{Fe}_{30}$ (top, left) and $\mathrm{Mo}_{132}$ (bottom, left) and a cartoon of their shell-like superstructure where the size range refers to the situation in aqueous solutions. The 12 fivefold symmetrical units (with their bipyramid centers of $\mathrm{MoO}_{7}$ ) are linked by 30 iron $\left(\mathrm{Mo}_{72} \mathrm{Fe}_{30}\right)$ or $\mathrm{Mo}_{2}^{\mathrm{V}}$ groups $\left(\mathrm{Mo}_{132}\right)$. The $\mathrm{Mo}_{2}^{\mathrm{V}}$ groups are in turn stabilized by bidentate acetate ligants (not shown). Note the binuclear linkers of $\mathrm{Mo}_{132}$ in comparison to the mononuclear linkers of $\mathrm{Mo}_{72} \mathrm{Fe}_{30}$. The cartoons in this figure are not to the scale. 
$\mathrm{Mo}_{72} \mathrm{Fe}_{30}$. These macromolecules were prepared as in [4,5], respectively. The detailed structure of these clusters can be visualized as a result of icosahedral disposition of 12 constituent $(\mathrm{Mo}) \mathrm{Mo}_{5}$ pentagonal building blocks along the $12 C_{5}$ axes of the icosahedral point group. The remaining 30 "linking" $C_{2}$ sites in turn connect the 12 pentagonal units. Evidently, in case of $\mathrm{Mo}_{132}$, these linkers are $\mathrm{Mo}_{2}$ whereas in the case of $\mathrm{Mo}_{72} \mathrm{Fe}_{30}$, those linkers are $\mathrm{Fe}^{\mathrm{III}}$. Note that there are slight uncertainties in the composition of the clusters, reflected in the " $c a$ " "..8/9," and "..." above. This is due to delocalization of these ligands in the crystal.

The number of dissociable groups on the surface of the POMs, that is, the maximum number of charges these macroions may carry in solution, is 42 in case of $\mathrm{Mo}_{132}$ and 30 for $\mathrm{Mo}_{72} \mathrm{Fe}_{30}$. For $\mathrm{Mo}_{132}$, charges occur due to dissociation of ionic groups. In case of $\mathrm{Mo}_{72} \mathrm{Fe}_{30}$, charges probably occur because of water ligands attached to $30 \mathrm{Fe}$ centers that tend to deprotonate (like a weak acid), leading to negatively charged clusters in solution. It will be shown later, cf. Table I, that the zeta potentials are close to $-2 k T$ so that because of charge renormalization, as discussed below Eq. (2), the number of unit charges on the POMs of radius $R \approx 1.3 \mathrm{~nm}$ in water cannot much exceed 4 .

For both systems, samples contain $\sim 0.1-1 \mathrm{mg} / \mathrm{mL}$ material. No salt has been added to any of the samples. In the case of $\mathrm{Mo}_{72} \mathrm{Fe}_{30}$, it is well known that formation of shells is extremely slow, in particular at room temperature [6]. Therefore, samples were heated to $50{ }^{\circ} \mathrm{C}$ while the size of the structures formed was monitored using Dynamic Light Scattering (DLS) at angles in between $35^{\circ}$ and $120^{\circ}$, until no change of size in time was detected over a period of one month. Another three months later, samples were doublechecked and in two of them corresponding to the lowest relative dielectric constant $\left(\mathrm{Mo}_{72} \mathrm{Fe}_{30}\right.$ in propanol and acetone), the presence of shells could not be verified, which led us to conclude that in these particular samples, the initially formed objects were metastable. The aqueous $\mathrm{Mo}_{132}$ systems with varying acetone content were left in an oven at $30^{\circ} \mathrm{C}$ for $6-7$ days, and the size of the shells was measured by DLS in a similar way as for the $\mathrm{Mo}_{72} \mathrm{Fe}_{30}$ systems.

Zeta potentials $(\Psi)$ were deduced from the mobilities of the shells measured by a commercial ZetaPals, Brookhaven Instruments. For details, refer to Ref. [3]. In

TABLE I. Values of measured zeta potential $(\psi)$, critical aggregation concentration (CAC), values of cohesive bond energies $u$ extracted from the slope in Fig. 2, and the interaction energy per bond $\Delta \mu^{0} / 3$ estimated from the CAC.

\begin{tabular}{lcc}
\hline \hline & $\mathrm{Mo}_{72} \mathrm{Fe}_{30}$ & $\mathrm{Mo}_{132}$ \\
\hline$\psi(m V)$ & $-56(p \mathrm{H}=4.6)$ & -48 \\
$\mathrm{CAC}(\mathrm{mg} / \mathrm{mL})$ & 0.0024 & $\cdots$ \\
$u(k T)$ & -6.8 & -5.4 \\
$\Delta \mu^{0} / 3(k T)$ & -6.6 & $\cdots$ \\
\hline \hline
\end{tabular}

case of the $\mathrm{Mo}_{72} \mathrm{Fe}_{30}$, in pure water, a significant $p \mathrm{H}$ dependence was observed: $\Psi=-47 \mathrm{mV}$ at $p \mathrm{H}=4.0$, while $\Psi=-70 \mathrm{mV}$ at $p \mathrm{H}=5.5$. The value of $\Psi$ for this POM listed in Table I corresponds to $p \mathrm{H}=4.6$ of a system with $0.1 \mathrm{mg} / \mathrm{mL}$ material, i.e., under the same condition as the other experiments with $\mathrm{Mo}_{72} \mathrm{Fe}_{30}$.

In Fig. 2, we plotted the size of the shells made of the two different POMs as determined by dynamic light scattering (extrapolated to zero angle) as a function of the inverse relative dielectric constant of the solvent medium. This way of plotting is in anticipation of the theory presented in this Letter that predicts a linear dependence between shell radius and inverse relative dielectric constant. The last quantity was varied by using different solvents or mixtures of them. In Table I, the measured values of the zeta potentials are listed.

We will now formulate a model to rationalize the observed sizes of the POM shells. We will presume that the driving force for the formation of the shells is pairwise additive attractive interaction between the POMs. We further assume that their equilibrium size is determined by their renormalized charge density, which in turn is being regulated by counterion condensation. This leads to the prediction that the size of the shells grows linearly with the inverse of the dielectric constant of the medium. This, indeed, is what we observe, and it points to a new stabili-

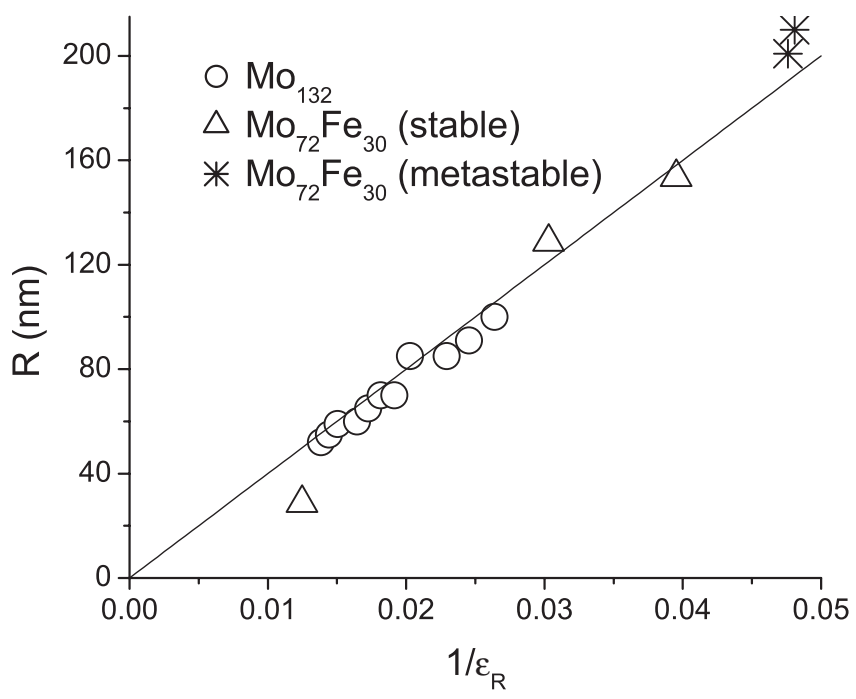

FIG. 2. Shell radius as determined by dynamic light scattering as a function of the inverse relative dielectric constant of the solvent for two different polyoxometalates: $\mathrm{Mo}_{72} \mathrm{Fe}_{30}$ and $\mathrm{Mo}_{132}$. The theory of this Letter suggests a linear relation between the radius and the inverse dielectric constant. Dielectric constants were varied by using acetone-water mixtures $\left(10-70 \%\right.$ in volume) in case of $\mathrm{Mo}_{132}$. For the samples with $\left(\mathrm{Mo}_{72} \mathrm{Fe}_{30}\right)$, the pure solvents water, methanol, ethanol, propanol, and acetone were used. Except for two points corresponding to $\left(\mathrm{Mo}_{72} \mathrm{Fe}_{30}\right)$ in pure propanol and acetone (as indicated; also see text), data correspond to thermodynamically stable shells. The slope of the line is given by $3824 \pm 266 \mathrm{~nm}$. 
zation mechanism for finite-size shells, which is referred to as charge regulation.

We assume the free energy $F$ of a shell depends on two fluctuating and dependent variables: its aggregation number, reflected in the radius $R$, and the effective charge $Z$,

$$
\frac{F}{k T}=4 \pi \gamma_{0} R^{2}+4 \pi(2 K+\bar{K})+\frac{\lambda_{B} Z^{2}}{2 R(1+\kappa R)}-\Psi Z .
$$

In this equation, $k$ denotes Boltzmann's constant, $T$ the absolute temperature, $\lambda_{B}=\frac{e^{2}}{4 \pi \epsilon_{0} \epsilon_{R} k T}$ the Bjerrum length, and $\kappa=\left(8 \pi \lambda_{B} \rho_{s}\right)^{1 / 2}$ the inverse Debye screening length, with $e$ the unit charge, $\epsilon_{0}$ the permittivity of vacuum, $\epsilon_{R}$ the relative dielectric constant of the medium, and $\rho_{s}$ the number density of the 1:1 electrolyte. The first term with surface tension $\gamma_{0}$ in Eq. (1) is extensive in the aggregation number and will not show up in the equilibrium equation, assuming the average area occupied by a POM monomer does not depend on R. The second term, with bending elastic modulus $K$ and Gaussian modulus $\bar{K}$, is related to the curvature contribution from the Helfrich expansion of a spherical vesicle [7]. The third and fourth terms, where $\Psi$ again denotes the zeta potential, regulate the effective charge of the aggregate. In fact, the third term arises from the screened-Coulomb interactions on a uniformly charged sphere in a background electrolyte characterized by a Debye screening length $\kappa^{-1}$, within the Debye-Hückel approximation, see [8]. This term is supposed to be correct as long as counter ions inside the shells can be neglected, being the case for $R \leq \kappa^{-1}$. The fourth term determines the extent of escape of ions from the narrow Gouy layer surrounding the shell. This term corresponds to a Legendre transformation from a constant charge-to a constant potential ensemble, see, e.g., [9]. Constant potential is an appropriate condition if the majority of counterions is confined to a narrow layer (the Gouy layer) around the surface [8]. In other words, the bare charge exceeds the effective charge by far. An additional condition is that the concentration of potential determining ions is constant. Potential determining ions in this case are those ions that escape from the Gouy layer leaving the surface to have a renormalized charge [10]. The absence of a term proportional to $R$ in Eq. (1) is consistent with the absence of dislocations [11]. It will be checked later if that assumption is justified.

The optimum renormalized charge of a shell with fixed $R$ follows from minimizing Eq. (1) with respect to $Z$, resulting in

$$
Z=\Psi \frac{R(1+\kappa R)}{\lambda_{B}} .
$$

This expression reduces to the cell-model result $Z=$ $\Psi\left(R / \lambda_{B}\right)$ in Ref. [10] if $\kappa R \ll 1$. Reported experimental values of $|\Psi|$ are close to $6 \mathrm{kT}$ for micelles [12] and highly charged colloids [13] in aqueous media, but also smaller values have been observed depending upon (bare) charge density and ionic strength $[8,14,15]$.
Inserting Eq. (2) into Eq. (1), the optimal cluster size is obtained by minimizing the free energy per unit area. This leads to

$$
R=\frac{16 \pi \lambda_{B}(2 K+\bar{K})}{\Psi^{2}} .
$$

As $\lambda_{B} \approx 56 / \epsilon_{R}$ nm, Eq. (3) predicts that $R \propto \epsilon_{R}^{-1}$. It is expected that the zeta potential $\Psi$ also depends on the dielectric constant, but only logarithmically, see Ref. [8] so that we expect the leading behavior given by Eq. (3). $\Psi$ also depends logarithmically on the concentration of potential determining ions, which are protons in case of $\mathrm{Mo}_{72} \mathrm{Fe}_{30}$ and ammonium ions in $\mathrm{Mo}_{132}$. Equation (2) implies that these concentrations vary with $R$, which in principle influences the value of $\Psi$. However, the variation of the concentration of potential determining ions caused by the variation in $R$ in the range shown in Fig. 2 is less than $1 \%$ of the total concentration of these ions. These total concentrations are significantly higher than the POM concentration, as excess acetic acid or ammonium acetate is present in the POM crystals from which the samples have been prepared. In Fig. 2, it is immediately verified that the size of the shells as a function of the inverse relative dielectric constant is indeed linear.

In order to illustrate the subtle yet crucial role of counter ion condensation, suppose the last, charge-regulating term was absent in Eq. (1). If that were the case, the charge density on a shell would be constant and the radius $R$ in Eq. (3) proportional to $\epsilon_{R}^{1 / 3}$.

The presence of a significant number of dislocations or "scars" in the shells, as observed in [16], would lead to a term proportional to $R$ in Eq. (1) [11]. This, in turn, results in a sublinear or even constant relation between $R$ and $\epsilon_{R}^{-1}$ in Fig. 2. Clearly this is not observed, and we conclude dislocations are absent as implicitly assumed in writing down Eq. (1). This is surprising, as our POM shells are significantly larger than the size where dislocations become obvious in [16].

We will now relate the curvature energy to the cohesive bond energy of the POMs on a shell. That energy will be compared later to the one extracted from the critical aggregate concentration. From Eulers theorem, it follows that for shells, independent of their size, at least 12 fivefold interacting monomers are required next to the predominantly present sixfold interactions. This implies that each shell misses at least 12 times the cohesive bond energy $u$ that monomer pairs have on the shell surface. Assuming this is the dominant contribution, in other words, $\bar{K} \gg K$, this translates into

$$
4 \pi(2 K+\bar{K}) \approx-12 u .
$$

Inserting this into Eq. (3), we finally obtain

$$
R \approx \frac{-48 \lambda_{B} u}{\Psi^{2}}
$$


The data for the two different polyoxometalates in Fig. 2 fall on the same line. Looking at Eq. (5), and the comparable values of the zeta potentials in Table I, this indicates that the cohesive energy per bond, $u$, is comparable for the two systems. From the slope in Fig. 2, and the values of $\Psi$ listed in Table I, we deduce cohesive bond energies $u \approx$ $-6.8 k T$ for $\mathrm{Mo}_{72} \mathrm{Fe}_{30}$ and $u \approx-5.4 k T$ for $\mathrm{Mo}_{132}$. Considering the uncertainty of the slope in Fig. 2 as well as the $\sim 10$ percent uncertainty in the values of $\Psi$, the values of $u$ of the two POMs are not significantly different. We will now compare the value of $u$ for $\mathrm{Mo}_{72} \mathrm{Fe}_{30}$ with the interaction energy deduced from an entirely independent quantity: the critical aggregation concentration (CAC). For $\mathrm{Mo}_{72} \mathrm{Fe}_{30}$, the CAC as obtained in [17] and listed in Table I is $0.0024 \mathrm{mg} / \mathrm{mL}$ (the CAC value for $\mathrm{Mo}_{132}$ is unknown). The CAC mole fraction is given by [18] $x_{\mathrm{CAC}}=$ $\exp \left(\Delta \mu^{0} / \mathrm{kT}\right)$, where $\Delta \mu^{0}$ is the difference in standard chemical potential between a POM in a shell and in solution. The molecular weight of $\mathrm{Mo}_{72} \mathrm{Fe}_{30}$ being $18000 \mathrm{~g} / \mathrm{mole}$, and assuming shells consist of predominantly hexagonally arranged polyoxymetals, it follows that $u \approx \Delta \mu^{0} / 3 \approx-6.6 k T$. This value is in excellent agreement with the value of $u$ obtained from our model and provides additional plausibility that the finite-size POM shells are indeed stabilized by charge regulation. The situation is summarized in Table I.

The values of the cohesive bond energy for the two POMs in Table I are consistent with hydrogen bonds [18] and also with the weak metal-oxygen bonds in simple complexes of Molybdenum(VI) oxide [19]. These values also are typical for those found in other self-assembling systems such as surfactant micelles [18] and virus capsids [20]. It is quite intriguing that the apparently metastable systems indicated in Fig. 2, i.e., the largest two shells, behave in line with the stable systems. This probably is a consequence of the slow dynamics in the system, which tends to be trapped for long times in its local free energy minimum before moving to its global equilibrium state.

We showed that the finite-size shells made of POMs at low ionic strengths are stabilized by charge regulation reflected in Eq. (5). In principle, this mechanism should also be operative in thermodynamically stable (charged) surfactant vesicles without added electrolyte, such as those studied in, e.g., [21-23]. While the origin of the attractive forces in these systems is different from the POM-shells, that should not lead to different behavior at this level of description. In Ref. [24], the measured sizes of so-called microvesicles without added salt are analyzed using a cell model. These microvesicles become unstable if even a small amount of salt is added. This points to the mechanism reported here: if $\kappa^{-1}<R$, the third term in Eq. (1) breaks down, implying objects other than "microvesicles" may become stable. At these higher ionic strengths, finitesize shells may become entropically stabilized [25]. In these systems, we would not expect the behavior dictated by Eq. (5).

We thank Paul van der Schoot for discussions.

*Corresponding authors: TIL204@lehigh.edu; w.k.kegel@uu.nl

[1] A. Müller and S. Roy, Coord. Chem. Rev. 245, 153 (2003).

[2] T. Liu, E. Diemann, H. Li, A. Dress, and A. Müller, Nature (London) 426, 59 (2003).

[3] G. Liu, T. Liu, S. S. Mal, and U. Kortz, J. Am. Chem. Soc. 128, 10103 (2006).

[4] A. Müller, S. Sarkar, S. Q. N. Shah, H. Bögge, M. Schmidtmann, S. Sarkar, P. Kögerler, B. Hauptfleisch, A.X. Trautwein, and V. Schünemann, Angew. Chem., Int. Ed. Engl. 38, 3238 (1999).

[5] A. Müller, E. Krickemeyer, H. Bögge, M. Schmidtmann, and F. Peters, Angew. Chem., Int. Ed. Engl. 37, 3360 (1998).

[6] T. Liu, J. Am. Chem. Soc. 125, 312 (2003).

[7] W. Helfrich, Z. Naturforsch. 28c, 693 (1973).

[8] R. J. Hunter, Foundations of Colloid Science (Oxford University Press, New York, 2001), 2nd ed..

[9] R. P. Feynman, R. B. Leighton, and M. L. Sands, The Feynman Lectures on Physics-Commemorative Issue (Addison-Wesley, Redwood CA, 1989), Vol. II.

[10] S. Alexander, P. M. Chaikin, P. Grant, G. J. Morales, and P. Pincus, J. Chem. Phys. 80, 5776 (1984).

[11] M. J. Bowick, D. R. Nelson, and A. Travesset, Phys. Rev. B 62, 8738 (2000).

[12] S. Bucci, C. Fagotti, V. Degiorgio, and R. Piazza, Langmuir 7, 824 (1991).

[13] J. C. Crocker and D. G. Grier, Phys. Rev. Lett. 77, 1897 (1996).

[14] E. Aicart, P. del Burgo, O. Llorca, and E. Junquera, Langmuir 22, 4027 (2006).

[15] F. Bordi, C. Cametti, S. Sennato, B. Paoli, and C. Marianecci, J. Phys. Chem. B 110, 4808 (2006).

[16] A. R. Bausch, M. J. Bowick, A. Cacciuto, A. D. Dinsmore, M. F. Hsu, D. R. Nelson, M. G. Nikolaides, A. Travesset, and D. A. Weitz, Science 299, 1716 (2003).

[17] G. Liu and T. Liu, J. Am. Chem. Soc. 127, 6942 (2005).

[18] J. Israelachvili, Intermolecular and Surface Forces (Academic Press, San Diego, 1992), 2nd ed..

[19] I. L. Validzic, G. vanHooijdonk, S. Oosterhout, and W. K. Kegel, Langmuir 20, 3435 (2004).

[20] W. K. Kegel and P. van der Schoot, Biophys. J. 86, 3905 (2004).

[21] M.M.A.E. Claessens, B.F. van Oort, F.A.M. Leermakers, F. A. Hoekstra, and M.A. Cohen Stuart, Biophys. J. 87, 3882 (2004).

[22] E.W. Kaler, A.K. Murthy, B.E. Rodriquez, and J. Zasadzinski, Science 245, 1371 (1989).

[23] B. Coldren, R. van Zanten, M. Mackel, J. Zasadzinski, and H.-T. Jung, Langmuir 19, 5632 (2003).

[24] J. Oberdisse and G. Porte, Phys. Rev. E 56, 1965 (1997).

[25] W. K. Kegel and H. Reiss, Ber. Bunsen-Ges. Phys. Chem. 100, 300 (1996). 